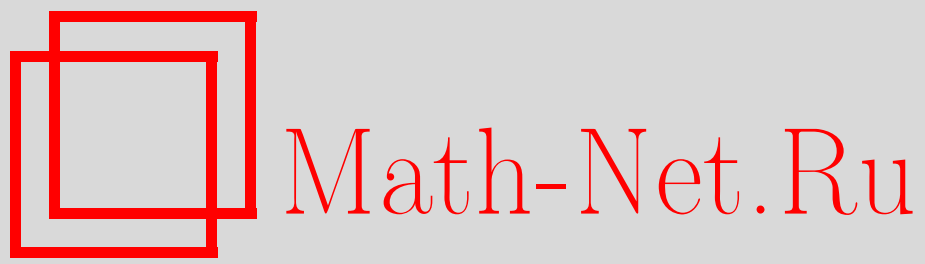

В. И. Жегалов, Об одной задаче для обобщенного уравнения Буссинеска-Лява, Вестн. Сам. гос. техн. ун-та. Сер. Физ.-мат. науки, 2019, номер 4, 771-776

DOI: https://doi.org/10.14498/vsgtu1720

Использование Общероссийского математического портала MathNet.Ru подразумевает, что вы прочитали и согласны с пользовательским соглашением

http://www . mathnet.ru/rus/agreement

Параметры загрузки:

IP : 54.197 .217 .227

26 апреля 2023 г., 14:08:21

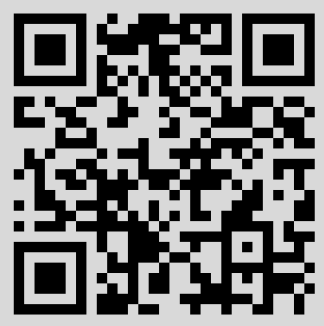


УДК 517.956

\title{
Об одной задаче для обобщенного уравнения Буссинеска-Лява
}

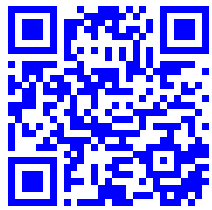

\section{В. И. Жегалов}

Казанский (Приволжский) федеральный университет,

Институт математики и механики им. Н. И. Лобачевского,

Россия, 420008, Казань, ул. Кремлевская, 35.

\begin{abstract}
Аннотация
Для уравнения четвертого порядка с двумя независимыми переменными рассматривается вариант задачи Гурса с данными на двух пересекающихся характеристиках, включающий в себя не только построение искомой функции, но и определение коэффициентов уравнения. Таким образом, речь идет об обратной задаче с определением коэффициентов уравнения. Предложена методика построения условий, обеспечивающих выделение бесконечного числа наборов уравнений данного вида, для которых рассматриваемая задача разрешима в квадратурах. Вместо введения дополнительных граничных условий предлагаются ограничения на структуру уравнения, связанные с возможностями его факторизации.
\end{abstract}

Ключевые слова: задача Гурса, обратная коэффициентная задача, метод каскадного интегрирования, метод Римана, факторизация.

Получение: 14 июля 2019 г. / Исправление: 28 октября 2019 г.

Принятие: 11 ноября 2019 г. / Публикация онлайн: 18 ноября 2019 г.

Указанное в названии сообщения уравнение Буссинеска-Лява имеет вид [1, формула (20)]

$$
u_{x x y y}-u_{y y}+u_{x x}=0 .
$$

Оно возникает при изучении движения волн в периодических слоистых средах [2], а также в упругом стержне с учетом эффектов поперечной инерции.

Нашей целью является отыскание условий разрешимости в квадратурах задачи Гурса для уравнения

$$
u_{x x y y}+a_{21} u_{x x} y+a_{12} u_{x y y}+a_{11} u_{x y}+a_{20} u_{x x}+a_{02} u_{y y}+a_{10} u_{x}+a_{01} u_{y}+a_{00} u=0,
$$

которое рассматривается в области $D=\left\{x_{0}<x<x_{1}, y_{0}<y<y_{1}\right\}$. Гранич-

\section{Краткое сообщение}

ə (ㅇ) Контент публикуется на условиях лицензии Creative Commons Attribution 4.0 International (https://creativecommons.org/licenses/by/4.0/deed.ru)

\section{Образец для цитирования}

$\mathrm{Z}$ hegalov V. I. On a proplem for generalized Boussinesq-Love equation, Vestn. Samar. Gos. Tekhn. Univ., Ser. Fiz.-Mat. Nauki [J. Samara State Tech. Univ., Ser. Phys. Math. Sci.], 2019, vol. 23 , no. 4, pp. 771-776. doi: 10.14498/vsgtu1720.

\section{Сведения об авторе}

Валентин Иванович Жегалов (1) https://orcid.org/0000-0002-3956-7026

доктор физико-математических наук, профессор; профессор; каф. математического анализа; e-mail: vizhegalov@mail.ru 
ные условия для (1) имеют при этом вид

$$
\begin{gathered}
u\left(x_{0}, y\right)=\varphi(y), \quad u_{x}\left(x_{0}, y\right)=\varphi_{1}(y) \\
u\left(x, y_{0}\right)=\psi(x), \quad u_{y}\left(x, y_{0}\right)=\psi_{1}(x), \\
\varphi^{\prime}\left(y_{0}\right)=\psi_{1}\left(x_{0}\right), \quad \varphi\left(y_{0}\right)=\psi\left(x_{0}\right), \\
\psi^{\prime}\left(x_{0}\right)=\varphi_{1}\left(y_{0}\right)
\end{gathered}
$$

при чем коэффициенты в (1) считаются неизвестными и вместе с $u(x, y)$ тоже подлежат определению.

С различных точек зрения уравнение (1) изучалось в работах Д. Манжерона, М. Огюсторели, С. Еасварана, В. Радочовы, Р. С. Жамалова, А. П. Солдатова и М. Х. Шханукова, Е. А. Уткиной (см. библиографию в книгах [3,4]), а также в работах $[5,6]$. В частности, в указанной монографии [3, с. 139-142] методом Римана построено решение задачи (1)-(2), но оно представляет собой лишь структурную формулу, поскольку используемая там функция Римана только существует, а конкретный вид ее неизвестен.

В данном сообщении изучается следующая

ЗАДачА. Определить коэбфиииенты уравнения (1), при которых его решение в области $D$, удовлетворяющее граничным условиям (2), оказывается разрешимым в квадратурах.

Имеется значительное количество работ, посвященных обратным коэффициентным задачам (см., например, работы [7-12] и литературу при них). Хорошо известен подход к определению в уравнении неизвестных коэффициентов, основанный на введении дополнительных граничных условий. Предлагаемый здесь подход к отысканию решения этой задачи основан на ограничениях, связанных с возможностями факторизации (1), то есть представлением этого уравнения либо в виде

$$
\left(\frac{\partial^{2}}{\partial x \partial y}+\alpha \frac{\partial}{\partial x}+\beta \frac{\partial}{\partial y}+\gamma\right)\left(u_{x y}+\lambda u_{x}+\mu u_{y}+\nu u\right)=0
$$

либо в форме, получаемой из (3) путем подстановки операторов (второй оператор ставится на первое место, а первый - на второе).

Производя в левой части (3) указанные действия, потребуем совпадения полученного выражения с коэффициентами из (1). Для (3) найдем

$$
\begin{gathered}
\alpha+\lambda=a_{21}, \quad \lambda_{y}+\alpha \lambda=a_{20}, \\
\mu+\beta=a_{12}, \quad \mu_{x}+\beta \mu=a_{02} ; \\
\lambda_{x}+\mu_{y}+\nu+\gamma+\mu \alpha+\beta \lambda=a_{11}, \\
\mu_{x y}+\nu_{x}+\beta\left(\mu_{y}+\nu\right)+\alpha \mu_{x}+\gamma \mu=a_{01}, \\
\lambda_{x y}+\nu_{y}+\alpha\left(\lambda_{x}+\nu\right)+\beta \lambda_{y}+\gamma \lambda=a_{10}, \\
\nu_{x y}+\alpha \nu_{x}+\beta \nu_{y}+\gamma \nu=a_{00} .
\end{gathered}
$$

Для уравнения, получаемого из (3) перестановкой операторов, нужно в левой части соотношений (4) функции $\alpha, \beta, \gamma$ заменить на $\lambda, \mu, \nu$, а функции $\lambda, \mu, \nu$ в (5) - на $\alpha, \beta, \gamma$.

Мы не будем выписывать формулы для (3) с переставленными операторами, так как порядок рассуждений не изменится.

Система (4), (5), как система для отыскания $\alpha, \beta, \gamma, \lambda, \mu, \nu$, является переопределенной (8 уравнений, 6 неизвестных). 
Сначала рассмотрим (4). Здесь первая пара уравнений - система для отыскания функций $\alpha, \lambda$. Подставляя во второе уравнение $\alpha=a_{21}-\lambda$, или $\lambda=a_{21}-\alpha$, получим уравнения Риккати

$$
\lambda_{y}+a_{21} \lambda-\lambda^{2}=a_{20}, \quad \alpha_{y}-a_{21} \alpha+\alpha^{2}=a_{20}-a_{21 y},
$$

которые в общем случае неразрешимы в квадратурах, но в случае нулевых правых частей являются уравнениями Бернулли, которые разрешимы в явном виде с точностью до произвольной функции от $x$.

Единственные решения выделяются при дополнительных условиях

$$
\alpha\left(x, y_{0}\right)=\xi(x), \quad \lambda\left(x, y_{0}\right)=\eta(x)
$$

где функции $\xi(x), \eta(x)$ должны быть заданы.

Аналогично вторая пара из (4) редуцируется к уравнениям

$$
\mu_{x}+a_{12} \mu-\mu^{2}=a_{02}, \quad \beta_{x}-a_{12} \beta+\beta^{2}=a_{02}-a_{12},
$$

а роль (6) играют формулы

$$
\beta\left(x_{0}, y\right)=\rho(y), \quad \mu\left(x_{0}, y\right)=\sigma(y) .
$$

Из изложенного вытекает

Лемма. В предположении, что

$$
a_{20} \equiv a_{02} \equiv a_{20} \equiv a_{20}-a_{21 y} \equiv a_{02}-a_{12 x} \equiv 0,
$$

и дополнителъных условиях (6), (7) функции $\alpha, \beta, \lambda, \mu$ однозначно определяются в квадратурах.

В условиях данной леммы уравнения (5) превращаются в формулы для определения $a_{11}, a_{01}, a_{10}, a_{00}$ через остающиеся пока неизвестными значениями $\gamma, \nu$, которые могут быть найдены путем подбора. А именно, взяв в качестве одной из них определенную функцию, другую найдем из первого уравнения (5). Остальные три соотношения (5) предлагается рассматривать как дополнительные требования, достаточные для однозначной разрешимости всей системы (4), (5) в явном виде. Поскольку $\gamma$ или $\nu$ можно фиксировать бесконечным числом способов, можно утверждать, что система (4), (5) допускает бесконечное число решений в достаточных для нас классах гладкости. Сведения об упомянутой гладкости можно найти в учебниках по дифференциальным уравнениям (например, в [13]).

Вышеизложенное позволяет представить рассматриваемую задачу в форме двух классических постановок задачи Гурса для уравнений второго порядка:

$$
\begin{gathered}
u_{x y}+\lambda u_{x}+\mu u_{y}+\nu u=v \\
u\left(x_{0}, y\right)=\varphi(y), u\left(x, y_{0}\right)=\psi(x), \varphi\left(y_{0}\right)=\psi\left(x_{0}\right) \\
v_{x y}+\alpha v_{x}+\beta v_{y}+\gamma v=0 \\
v\left(x_{0}, y\right)=\theta(y), v\left(x, y_{0}\right)=\theta_{1}(x), \theta\left(y_{0}\right)=\theta_{1}\left(x_{0}\right)
\end{gathered}
$$

где функции $\theta(y), \theta_{1}(x)$ в силу (2) легко вычисляются. Очевидно, что сначала находится решение задачи (9), а затем - задачи (8). 
Известен целый ряд вариантов разрешимости подобных задач в квадратурах, записываемых в терминах коэффициентов уравнений. Например, работы [3, с. 19-20], [14,15] содержат случаи, общее число вариантов разрешимости которых равно 16.

\section{Из приведенных рассуждений следует}

Теорема. Предложенная методика позволяет выделить бесконечное множество вариантов разрешимости рассматриваемой задачи в квадратурах.

Конкурирующие интересы. Конкурирующих интересов не имею.

Авторская ответственность. Я несу полную ответственность за предоставление окончательной версии рукописи в печать. Окончательная версия рукописи мною одобрена.

\section{Библиографический список}

1. Солдатов А. П., Шхануков М. Х. Краевые задачи с общим нелокальным условием А. А. Самарского для псевдогиперболических уравнений высокого порядка // Докл. AH CCCP, 1987. Т. 297, № 3. С. 547-552.

2. Сердюкова С. И. Экзотическая асимптотика для линейного гиперболического уравнения // Докл. РАН, 2003. Т. 389, № 3. С. 305-309.

3. Жегалов В. И., Миронов А. Н. Дифференииальные уравнения со стариими частными производными. Казань: Казанск. матем. об-во, 2001. 226 с.

4. Жегалов В. И., Миронов А. Н., Уткина Е. А. Уравнения с доминирующей частной производной. Казань: Казанск. ун-т, 2014. 385 с.

5. Миронов А. Н. О методе Римана решения задачи Коши // Изв. вузов. Матем., 2005. № 2. C. 34-44.

6. Миронов А. Н., Миронова Л. Б. Об инвариантах Лапласа для обобщенного уравнения Буссинеска-Лява // Диффер. уравн., 2015. Т.51, №1. С. 131-135. doi: 10.1134/ S0374064115010136.

7. Anikonov Yu. E., Belov Yu. Ya. Determining two unknown coefficients of parabolic type equation // J. Inverse Ill-posed Probl., 2001. vol. 9, no.5. pp. 469-487. doi: 10.1515/jiip. 2001.9 .5 .469 .

8. Anikonov Yu. E. Inverse problems and classes of solutions of evolution equations // J. Inverse Ill-posed Probl., 2003. vol. 11, no. 1. pp. 1-26. doi: 10.1515/156939403322004900.

9. Алексеев Г. В., Вахитов И. С., Соболева О. В. Оценки устойчивости в задачах идентификации для уравнения конвекции-диффузии-реакции // Ж. вычисл. матем. и матем. физ., 2012. Т. 52, № 12. С. 2190-2205.

10. Камынин В. Л. Обратная задача одновременного определения правой части и младшего коэффициента в параболическом уравнении со многими пространственными переменными // Матем. заметки, 2015. Т. 97, № 3. С. 368-381. doi : 10.4213/mzm10499.

11. Кожанов А. И. Параболические уравнения с неизвестными коэффициентами, зависящими от времени // Ж. вычисл. матем. и матем. физ., 2017. Т. 57, №6. С. 961-972. doi: $10.7868 / \mathrm{S} 0044466917060096$.

12. Сабитов К. Б. Начально-граничная и обратные задачи для неоднородного смешанного параболо-гиперболического уравнения // Матем. заметки, 2017. Т. 102, №3. С. 415435. doi : 10.4213/mzm11521.

13. Сабитов К. Б. Функциональные, дифференциальные и интегральные уравнения. М.: Высш. шк., 2005. 670 с.

14. Жегалов В. И., Сарварова И. М. К условиям разрешимости задачи Гурса в квадратуpax // Изв. вузов. Матем., 2013. № 3. С. 68-73.

15. Жегалов В. И., Созонтова Е. А. Дополнение к случаям разрешимости задачи Гурса в квадратурах// Диффер. уравн., 2017. Т. 53, №2. С. 270-273. doi:10.1134/ S0374064117020133. 
MSC: 35L25

\title{
On a proplem for generalized Boussinesq-Love equation
}

\section{I. Zhegalov}

Kazan Federal (Volga Region) Federal University,

N. I. Lobachevsky Institute of Mathematics and Mechanics,

35, Kremlyovskaya str., Kazan, 420008, Russian Federation.

\begin{abstract}
For a fourth-order equation with two independent variables a variant of the Goursat problem with data on two intersecting characteristics is considered. It includes not only the construction of the desired function, but also the coefficients of the equation. Thus, we are talking about the inverse problem of determining the coefficients of the equation. The method of construction of conditions providing allocation of infinite number of sets of this type equations is offered, for which the problem under consideration is solvable in quadratures. Instead of introducing additional boundary conditions, restrictions on the structure of the equation are proposed, related to the possibilities of its factorization.
\end{abstract}

Keywords: Goursat problem, inverse problem, method of cascade integration, Riemann method, factorization.

Received: $14^{\text {th }}$ July, 2019 / Revised: $28^{\text {th }}$ October, $2019 /$

Accepted: $11^{\text {th }}$ November, $2019 /$ First online: $18^{\text {th }}$ November, 2019

Competing interests. I declare that I have no competing interests.

Author's Responsibilities. I take full responsibility for submitting the final manuscript in print. I approved the final version of the manuscript.

\section{References}

1. Soldatov A. P., Shkhanukov M. Kh. Boundary-value-problems with a Samarsky, A. A. general nonlocal condition for higher-order pseudoparabolic equations, Dokl. Math., 1988, vol. 36, no. 3, pp. 507-511.

2. Serdyukova S. I. Exotic asymptotics for a linear hyperbolic equation, Dokl. Math., 2003, vol. 67, no. 2, pp. 203-207.

\section{Short Communication}

()(i) The content is published under the terms of the Creative Commons Attribution 4.0 International License (http://creativecommons.org/licenses/by/4.0/)

Please cite this article in press as:

Zhegalov V. I. On a proplem for generalized Boussinesq-Love equation, Vestn. Samar. Gos. Tekhn. Univ., Ser. Fiz.-Mat. Nauki [J. Samara State Tech. Univ., Ser. Phys. Math. Sci.], 2019, vol. 23, no. 4, pp. 771-776. doi: 10.14498/vsgtu1720.

\section{Author's Details:}

Valentin I. Zhegalov (1) https://orcid.org/0000-0002-3956-7026

Dokt. Phys. \& Math. Sci.; Professor; Dept. of Mathematical Analisys;

e-mail: vizhegalov@mail.ru 
3. Zhegalov V. I., Mironov A. N. Differentsial'nye uravneniia so starshimi chastnymi proizvodnymi [Differential Equations with Highest Partial Derivatives]. Kazan, Kazan Math. Society, 2001, 226 pp. (In Russian)

4. Zhegalov V. I., Mironov A. N., Utkina E. A. Uravneniia s dominiruiushchei chastnoi proizvodnoi [Equations with Dominant Partial Derivative]. Kazan, Kazan Univ., 2014, 385 pp. (In Russian)

5. Mironov A. N. On the Riemann method for solving the Cauchy problem, Russian Math. (Iz. VUZ), 2005, vol. 49, no. 2, pp. 32-41.

6. Mironov A. N., Mironova L. B. Laplace invariants for a generalized Boussinesq-Love equation, Differ. Equ., 2015, vol. 51, no. 1, pp. 132-137. doi: 10.1134/S0012266115010139.

7. Anikonov Yu. E., Belov Yu. Ya. Determining two unknown coefficients of parabolic type equation, J. Inverse Ill-posed Probl., 2001, vol.9, no. 5, pp. 469-487. doi:10.1515/jiip. 2001.9.5.469.

8. Anikonov Yu. E. Inverse problems and classes of solutions of evolution equations, J. Inverse Ill-posed Probl., 2003, vol. 11, no. 1, pp. 1-26. doi : 10.1515/156939403322004900.

9. Alekseev G. V., Vakhitov I. S., Soboleva O. V. Stability estimates in identification problems for the convection-diffusion-reaction equation, Comput. Math. Math. Phys., 2012, vol.52, no. 12, pp. 1635-1649. doi: 10.1134/S0965542512120032.

10. Kamynin V. L. The inverse problem of the simultaneous determination of the right-hand side and the lowest coefficient in parabolic equations with many space variables, Math. Notes, 2015, vol.97, no. 3, pp. 349-361. doi: 10.1134/S0001434615030062.

11. Kozhanov A. I. Parabolic equations with unknown time-dependent coefficients, Comput. Math. Math. Phys., 2017, vol.57, no.6, pp. 956-966. doi: 10.1134/S0965542517060082.

12. Sabitov K. B. Initial boundary and inverse problems for the inhomogeneous equation of a mixed parabolic-hyperbolic equation, Math. Notes, 2017, vol.102, no.3, pp. 378-395. doi : 10.1134/S0001434617090085.

13. Sabitov K. B. Funktsional'nye, differentsial'nye $i$ integral'nye uravneniia [Functional, Differential and Integral Equations]. Moscow, Vysshaya Shkola, 2005, 670 pp. (In Russian)

14. Zhegalov V. I., Sarvarova I. M. Solvability of the Goursat problem in quadratures, Russian Math. (Iz. VUZ), 2013, vol.57, no. 3, pp. 56-59. doi: 10.3103/S1066369X13030080.

15. Zhegalov V. I., Sozontova E. A. An addition to the cases of solvability of the Goursat problem in quadratures, Differ. Equ., 2017, vol.53, no.2, pp. 270-272. doi: 10.1134/ S0012266117020124. 